\title{
Riesgo e Ideación Suicida y su Relación con la Impulsividad y la Depresión en Adolescentes Escolares
}

\author{
Risk and Suicidal Ideation and its Relationship with Impulsivity and Depression in \\ School Adolescent
}

\author{
Anyerson Stiths Gómez Tabares ${ }^{1}$, César Núñez ${ }^{2}$, María Paula Agudelo Osorio ${ }^{3}$ \\ y Andrés Mauricio Grisales Aguirre ${ }^{4}$
}

\begin{abstract}
Resumen
Por medio de un diseño transversal de alcance explicativo, se analizó la relación estadística entre riesgo suicida (RS) e ideación suicida (IS) con la depresión e impulsividad. Participaron 179 adolescentes escolarizados entre 14 a 17 años $(M=14.84 ; D E=0.82)$. Se utilizó la Escala de Riesgo Suicida de Plutchik (RS), el Inventario de Ideación Suicida Positiva y Negativa (PANSI), Inventario de Depresión de Beck (BDII) y la Escala de Impulsividad de Plutchik (EI). Se encontró un factor de riesgo suicida del $20.7 \%$ y correlaciones estadísticamente significativas $(p<.001)$, de signo positivo, entre RS, IS negativa y global, depresión e impulsividad. La IS positiva considerada como factor protector mostró correlación negativa con el RS, IS negativa y global, depresión e impulsividad. El análisis con ecuaciones estructurales mostró la depresión como variable mediadora entre el RS, la IS y la impulsividad $\left(\chi^{2}(2)=3.1 ; p=.212\right.$; CFI $=.985$; $\mathrm{NFI}=.963 ; \mathrm{TLI}=.926 ; \mathrm{GFI}=.987$; RMSEA=.056).
\end{abstract}

Palabras clave: Ideación suicida, riesgo, impulsividad, depresión, adolescente

\begin{abstract}
A non-experimental cross-sectional design and explanatory study were proposed in order to analyze the statistical relationship between the suicide risk (SR) and suicidal ideation (SI) and the depression and impulsivity variables in schooling adolescents. 179 subjects $(M=14.84 ; S D=0.82)$ between 14 and 17 years old participated. Variables were measured using the Plutchik Suicide Risk Scale (SRS), the Positive and Negative Suicide Ideation (PANSI) Inventory, the Beck Depression Inventory (BDI-I), and the Plutchik's Impulsivity Scale (IS). The results show a suicide risk factor of $20.7 \%$. Moreover, significant positive correlations $(p<.001)$ were found among SR, negative and global SI, depression and impulsivity. The positive SI considered as a protective factor showed a negative correlation with respect to SR, the negative and global SI, depression and impulsivity. The structural equation modeling analysis applied showed depression is the mediator variable between SR, SI and impulsivity $\left(\chi^{2}(2)=3.1 ; p=.212\right.$; CFI=.985; NFI=.963; TLI=.926; GFI=.987; RMSEA=.056).
\end{abstract}

Keywords: Suicidal ideation, risk, impulsivity, depression, adolescent

Financiación: Trabajo financiado por la Universidad Católica Luis Amigó, Medellín, Colombia, y con alianza de cooperación en producción científica de la Universidad de Medellín, Colombia

\footnotetext{
${ }^{1}$ Profesor Investigador Adscrito al Programa de Psicología. Facultad de Psicología y Ciencias Sociales, Universidad Católica Luis Amigó. http://orcid.org/0000-0001-7389-3178 , carrera 22 N 67A - 49, Manizales, Colombia. Tel.: (6)8815536. Correo: anyerspn.gomezta@amigo.edu.co

${ }^{2}$ Profesor Investigador Programa de Psicología. Universidad de Medellín, Medellín. http://orcid.org/0000-0001-8925-993X; carrera. 87 30-65, código postal 050026, Medellín, Colombia. Tel.: (4)3405481. Correo: cnunez@udem.edu.co.

${ }^{3}$ Joven Investigadora. Grupo de investigación en Psicología y Procesos Clínico-Sociales, Programa de Psicología, Universidad de Medellín, carrera. 87 30-65, código postal 050026, Medellín, Colombia. Tel.: (4)3405481. Correo: m.pao325@gmail.com

4 Profesor Investigador en el área de Ciencias Básicas - Matemáticas y Estadística, Universidad Católica Luis Amigó. https://orcid.org/0000-0002-4385-4474; carrera $22 \mathrm{~N}^{\circ}$ 67A - 49, Manizales, Colombia. Tel.: (6)8815536. Correo: andres.grisalesag@amigo.edu.co

Revista Iberoamericana de Diagnóstico y Evaluación - e Avaliação Psicológica. RIDEP · №54 · Vol.1 · 147-163 · 2020

ISSN: 1135-3848 print /2183-6051online
} 


\section{Introducción}

El suicidio es un fenómeno complejo y multicausal de grandes magnitudes e impactos a nivel mundial, en la actualidad alcanza altas tasas de mortalidad que afecta con mayor frecuencia a la población adolescente y joven. Debido a su impacto negativo en la sociedad, se ha considerado como un problema de salud pública que debe ser estudiado y abordado de manera interdisciplinar e intersectorial. De acuerdo con la Organización Mundial de la Salud (OMS), se estima que al año cerca de 800.000 personas se quitan la vida y muchas más intentan hacerlo; en el año 2015 fue la segunda causa principal de defunción en el grupo etario de 15 a 29 años de edad en todo el mundo (OMS, 2017). De acuerdo con Delgado et al., (2017), el suicidio representa el $1.4 \%$ de muertes a nivel mundial, encontrándose en las principales causas de muerte violenta.

Según el Instituto Nacional de Medicina Legal y Ciencias Forenses (Forensis, 2016), en Colombia el suicidio ha incrementado considerablemente en los últimos años. En las estadísticas presentadas en los últimos 10 años han ocurrido 19.177 casos, esto quiere decir 193 suicidios al mes y 6 suicidios por día. En el año 2016 se reportaron 2.310 suicidios, con un incremento de 242 casos (10.4\%) respecto del año anterior. Este incremento se ha presentado en las edades de 20 a 24 años (270 casos) y entre los 25 a 29 años (228 casos) en la población masculina, mientras que en la población femenina entre los 15 a 17 años (71 casos) y 20 a 24 años (57 casos), siendo mayor en hombres por la letalidad en los métodos que utilizan para suicidarse.

De acuerdo con la Dirección Territorial de Salud de Caldas (2016), en Colombia el suicidio es el tipo de violencia más frecuente con un incremento en el tiempo; para el año 2013 fue de 3.84 por cada 100.000 habitantes. Entre tanto para el 2016 en el departamento de Caldas tuvo una tasa de 6.57 casos por cada 100.000 habitantes ocupando el sexto lugar de los departamentos con más suicidios en Colombia, ya que la tasa nacional para este momento fue de 3.76 suicidios por cada 100.000 habitantes. Para el año 2017 se registraron 15 suicidios entre enero y mayo y 368 intentos de suicidios, sin dejar de lado que durante el año 2016 se presentaron 65 suicidios en el departamento ya anotado (Delgado et al., 2017).

Por ciclo de desarrollo vital, en Caldas, durante el año 2016, el grupo etario con mayor número de caso de suicidios fue los adolescentes entre 15 a 19 años, con 11 casos (16.92\%), y jóvenes entre 20 a 24 años con 7 casos (10.77\%) y entre 25 a 29 años con 11 casos (19.92\%) (Delgado et al, 2017). El mismo autor señala que la proporción entre casos de suicidios e intentos de suicidio en Caldas muestra que, en los años 2014, 2015 y 2016 el número de casos reportados de muertes auto infringidas fue de 54, 73 y 65 respectivamente, mientras que la proporción de casos de intentos fue de 773, 981 y 770 respectivamente, lo cual indica que por cada suicidio consumado hay aproximadamente 13 personas que lo intentan.

Teniendo en cuenta los datos epidemiológicos mencionados en relación a este fenómeno, se considera importante, no solo contemplar el suicidio consumado, sino, especialmente, la ideación, el riesgo y el intento suicida, dado que el espectro suicida debe entenderse como un proceso complejo y escalonado que inicia con la representación y los pensamientos de muerte antes de que se dé cualquier intento (Gvion \& Apte, 2011; Fernández-Liporace \& Casullo, 2006). Generalmente una persona en riesgo puede presentar ideas persistentes de suicidio, considerar la muerte como una opción válida y deseable, emergen pensamientos automáticos e intrusivos de que la vida carece de sentido, muchas veces acompañado de fantasías auto destructivas hasta llegar a planes estructurados de muerte (Gutiérrez, Contreras, \& Rodríguez, 2006; Carmona et al., 2017; Witte et al., 2008); la ideación suicida previa al evento conductual del intento de suicidio es un predictor significativo (Mortier et al., 2017).

La ideación suicida y los factores de riesgo psicosocial se han considero una prioridad para la investigación en ciencias sociales. Diversos estudios en Colombia (Fuentes et al., 2009; Villalobos, 2009; Siabato \& Salamanca, 2015), muestran que la prevalencia más alta de intentos de suicidio se presenta en población adolescente y joven, especialmente entre los 15 a 24 años de edad. Esta tendencia también se ha encontrado en estudios en Caldas (Álvarez et al., 2013; Cañón et al., 2012, Carmona \& Carmona, 2014; Castaño et 
al., 2015; Amézquita, González, \& Zuluaga, 2003, 2008; Fuentes et al., 2009), donde ubican este grupo etario en un mayor nivel de riesgo para el suicidio en comparación a otras etapas de la vida.

Esta tendencia se debe a la exposición a estresores psicosociales de diversa índole que, sumado a factores de vulnerabilidad psicosocial (Madge et al., 2017) como lo es la baja tolerancia a la frustración, la impulsividad, la depresión y la falta de recursos psicológicos para manejar y solucionar problemas, juegan un papel importante en la ideación y el intento suicida en la adolescencia (Siabato \& Salamanca, 2015; Mortier et al., 2018).

Estos hallazgos son coherentes con los encontrados por Amezquita et al., (2008), Álvarez et al. (2013), Aguirre et al. (2014), Sánchez, Guzmán y Cáceres (2005), y Cañón et al. (2012), en el sentido de considerar que los adolescentes son la población con mayor riesgo e ideación suicida en comparación a otras etapas de la vida, encontrándose que factores como la depresión, problemáticas psicosociales, consumo de drogas y alcohol, disfuncionalidad familiar, baja tolerancia a la frustración, impulsividad aumentan el riesgo de suicidio y son predictores de la ideación suicida (Madge et al., 2011). Estos factores generan un malestar psicológico manifestándose en sentimientos de soledad, confusión, desesperanza y minusvalía, aumentando la posibilidad de presentar ideas suicidas (Amezquita et al., 2008; Franklin et al., 2017).

En cuanto al género, otros estudios realizados en Colombia (Villalobos, 2009a; Fuentes et al., 2009; Gómez et al., 2002; Salvo \& Melipillán, 2008; Rueda, Rangel, Castro, \& Camacho, 2010; Siabato, \& Salamanca, 2015), muestran que la ideación y el intento de suicidio se presenta con más frecuencia en mujeres que en hombres, mientras que los hombres tienen mayor riesgo al suicidio consumado, esto se debe, entre muchos factores, a la letalidad de los métodos utilizados, el número de intentos previos $\mathrm{y}$ factores psicológicos asociados, aspecto que es coincidente en estudios similares (Park \& Jang, 2018). Es importante considerar que una parte importante de investigaciones han examinado diferencias culturales y de género respecto de factores de riesgo de comportamiento suicida de forma aislada (Langhinrichsen-Rohling, Friend, \& Powell, 2009).

Oquendo (2010) y Villalobos (2009a) han determinado que variables como la depresión y la impulsividad se relacionan de manera directa con la ideación suicida en la adolescencia. De acuerdo a Salvo y Melipillán (2008) la impulsividad tiene una relación directa con la suicidalidad. Para Téllez y Forero (2006) "La impulsividad es un factor que desinhibe el comportamiento y origina conductas de alto riesgo e incluso comportamientos suicidas, razón por la cual se asocia con intentos fallidos de suicidio o gestos suicidas" (p.67), de tal modo que, una alta impulsividad es un factor de riesgo para el intento de suicidio, especialmente en la adolescencia (Amezquita et al., 2008).

La impulsividad está asociada a un déficit en el procesamiento cognitivo y un pobre control inhibitorio, que en esta etapa del desarrollo se manifiesta con comportamientos irreflexivos, temerarios, explosivos y sin mediar las consecuencias de los actos, lo cual se convierte en un factor de riesgo y vulnerabilidad para el suicidio (Riaño-Hernández, Guillen, \& BuelaCasal, 2015; Oquendo, 2010; Dumais et al., 2005; Gonzales et al., 2015) y ha sido asociado con trastornos limítrofes de personalidad (Carpiniello, Lai, Pirarba, Sardu, \& Pinna, 2011). En este sentido, de acuerdo a Castañeda (2016), un déficit en el funcionamiento ejecutivo está relacionado con el riesgo y el comportamiento suicida en general, justamente porque puede encaminar al aumento de la impulsividad, la hostilidad, la irreflexibilidad cognitiva y todo tipo de comportamientos autodestructivos que pueden acrecentarse ante eventos vitales estresantes, tal como lo muestran Auerbach, Stewart y Johnson (2017) y Alasaarela, Hakko, Riala y Riipinen (2017).

De otro lado, el soporte empírico en diversos estudios (Coffin, Álvarez, \& Marín, 2011; Siabato, \& Salamanca, 2015; Siabato, Forero, \& Salamanca, 2017; Villalobos-Galvis, 2009b; Amezquita et al., 2008; Arenas-Landgrave, LucioGómez Marqueo, \& Forns, 2012) muestran que la depresión es uno de los principales predictores de la ideación suicida en población adolescente y joven, a su vez, tanto la sintomatología depresiva como el cuadro clínico, especialmente la 
depresión mayor, son los principales factores que explican la ideación y el riesgo suicida. Según la OMS (2017), la depresión puede llegar a hacerse crónica o recurrente, y dificultar sensiblemente el desempeño laboral, escolar o familiar y en la capacidad para afrontar la vida diaria, aspectos que permitan explicar cómo en su forma más grave, puede conducir al suicidio (Croarkin et al., 2018; Miché et al., 2018).

Tanto la depresión como la impulsividad son variables que están estrechamente relacionadas con el riesgo y la ideación suicida en la adolescencia, sin embargo, el estudio de estos dos constructos se ha tomado de manera aislada (Orri, Galera, Turecki, Forte, Renaud, \& Boivin et al., 2018), o en su defecto, se ha puesto mayor atención al estudio de la depresión en relación a la conducta suicida (Franklin et al., 2017; Park, \& Jang, 2018). En este sentido, se considera importante profundizar en el estudio de las relaciones entre riesgo e ideación suicida con la depresión y la impulsividad en adolescentes escolares, lo cual podría orientar el establecimiento de estrategias focalizadas y contextualizadas en la prevención del suicidio que respondan a necesidades, no solo a nivel psicológico, sino también a nivel social y cultural en esta población.

De acuerdo a la revisión de antecedentes y el contexto epidemiológico presentado, éste estudio muestra la relación entre el riesgo y la ideación suicida en la adolescencia, especialmente en el papel que tiene la impulsividad y la depresión como factores importantes de vulnerabilidad en esta etapa del desarrollo.

\section{Método}

Se trata de una investigación cuantitativa, con diseño no experimental de tipo transversal. De acuerdo a Hernández Sampieri, Collado y Baptista (2014), las investigaciones cuantitativas de diseño no experimental se caracterizan por no realizar manipulación intencional y deliberada de las variables, su objetivo es estudiar un fenómeno en su contexto natural para realizar análisis posteriores. Es transversal en el sentido que se realizaron mediciones en un único momento. El alcance es explicativo en cuanto se centra en explicar los factores psicológicos asociados al comportamiento suicida en la muestra de escolares.

\section{Población y muestra}

La muestra estuvo compuesta por 179 adolescentes entre 14 a 17 años $(M=14.84$; $D E=.82)$, los cuales se encuentran en los grados noveno $(\mathrm{n}=99)$ y décimo $(\mathrm{n}=80)$. El $60.3 \%$ corresponde al género femenino y el $39.7 \%$ al género masculino. El $92 \%$ son de estratos 2 y 3 . En cuanto a la constitución del núcleo familiar, el $40.2 \%$ provienen de familias de tipo nuclear, el $34.1 \%$ de familia monoparental, el $23.5 \%$ de familia extensa y el $2.2 \%$ de familia mixta. El rango de edad para esta muestra se seleccionó teniendo en cuenta que la tasa más alta de suicidio se reporta en adolescentes entre 14 a 18 años según el observatorio social de la dirección territorial seccional Caldas (2017).

\section{Instrumentos}

Escala de riesgo suicida de Plutchik (RS). Es una escala tipo Likert diseñada por Plutchik (Plutchik \& van Praag, 1989), que busca diferenciar las personas que presentan algún riesgo de presentar un intento de suicidio de los que no. La escala se compone de 15 ítems cuyas opciones de respuesta son de $\mathrm{Si}$ o No, cada respuesta afirmativa suma un punto, para un total de 15; a mayor puntaje mayor es el riesgo suicida. La prueba presenta una consistencia de interna de .90 , fiabilidad tes-retest de .89 , sensibilidad y especificidad de $88 \%$ para un punto de corte en 6 (Rubio et al., 1998). En Colombia y Caldas se ha utilizado en diversos estudios con población de adolescentes y jóvenes (Aguirre et al., 2014; Álvarez et al., 2013; Fuentes et al., 2009), mostrando una buena consistencia interna. Para este estudio se realizó un análisis de fiabilidad con alpha de Cronbach (version equivalente al coeficiente 20 de Kuder - Richardson) evidenciando un coeficiente de .77 .

\section{Inventario de Ideación Suicida Positiva y} Negativa (PANSI). Es un cuestionario diseñado por Osman et al. (2002), que consta de 14 ítems, 6 de ideación suicida positiva (factores protectores) y 8 de ideación suicida negativa (factores de riesgo), evalúa la frecuencia en que el individuo 
ha tenido alguno de los 14 pensamientos en las últimas dos semanas. Su escala es de 5 puntos que oscilan entre 0 (nunca) a 4 (siempre). Este inventario fue validado por Villalobos $\left(2009^{\mathrm{b}}\right.$, 2010) en estudiantes de colegios y universidades de Colombia, mostrando una fiabilidad alta tanto para la escala total, con un alpha de Cronbach de .89 , como las sub escalas de ideación negativa (alpha de Cronbach de .91) e ideación positiva (alpha de Cronbach de .83). Para este estudio se realizó un análisis de fiabilidad con alpha de Cronbach evidenciando un coeficiente de .883 para la ideación suicida negativa y de .782 para la ideación suicida positiva.

Inventario de Depresión de Beck (BDI-I). Es una escala tipo likert diseñada por Beck, Rush, Shaw y Emery (1979), mide la presencia y gravedad de la depresión en adultos y adolescentes. Se compone de 21 ítems que evalúan la severidad de los síntomas cognitivos, afectivos, conductuales y fisiológicos de la depresión en un marco temporal de la última semana. Cada ítem se responde en una escala de 0 a 3. Las puntuaciones van de 0 y 63. Los puntos de corte son: 0-9, depresión leve o mínima; 10-16, depresión leve; 17-29, depresión moderada; y 3063, depresión grave. Posee coeficientes de fiabilidad de consistencia interna de .83 , con coeficientes de fiabilidad test-retest que oscilaban entre .60 y .90 en la validación española (Sanz \& Vásquez, 1998), el inventario ha sido utilizado en diversas investigaciones en Colombia y Manizales (Ceballos, et al., 2015; Amezquita et al., 2008, 2003; Alonso Polo et al., 2015; Molina et al., 2018), mostrando ser un instrumento con una alta fiabilidad en población adolescente. Para este estudio se realizó un análisis de fiabilidad con alpha de Cronbach evidenciando un coeficiente de .90 .

La Escala de Impulsividad de Plutchik (EI). Es una escala diseñada originalmente por Plutchik \& Van Praag (1989), validada al español por Rubio et al., (1999). Es una escala tipo likert diseñada para evaluar la impulsividad relacionada con la agresividad y la conducta suicida. Se compone de 15 ítems con una escala de respuesta que se puntúa de 0 a 3 (nunca, a veces, a menudo, casi siempre). Los ítems 4, 6, 11 y 15 se puntúan en sentido inverso, con una puntuación que va de 0 a
45 puntos, se considera el punto de corte a partir de 20 puntos en la adaptación española, con una sensibilidad del $60 \%$ y una especificidad del $74 \%$. La consistencia interna de la escala de acuerdo al coeficiente de fiabilidad alpha de Cronbach fue .90 y la fiabilidad test-retest por el coeficiente de Correlación de Pearson fue de .91 (Rubio et al, 1999). Ha sido validada en población de habla Hispana en Salvador y México (Alcázar, Bouso, $\&$ Verdejo, 2015). Para este estudio se realizó un análisis de fiabilidad con alpha de Cronbach evidenciando un coeficiente de .79 .

\section{Consideraciones Éticas}

En consideración con ley 1090 de 2006 y la resolución 008430 de 1993, esta investigación obedece a los principios éticos de respeto, intimidad y dignidad, asegurando la confidencialidad y el anonimato de los participantes, tal y como se establece en el artículo 26 y 50 . Es importante indicar que se contó con el aval institucional, el consentimiento informado de los padres y el asentimiento de los participantes, de igual manera se hizo un proceso de devolución de los resultados obtenidos a nivel institucional.

\section{Procedimiento}

La información de la presente investigación se recolectó durante el $1^{\circ}$ semestre del año 2018 con aval institucional, consentimiento informado de padres de familia y el asentimiento de los adolescentes participantes en el estudio. La aplicación de los instrumentos se realizó de manera colectiva en las aulas de clase de los grados noveno y décimo de acuerdo al rango de edad de interés, previo a la explicación del objetivo de la investigación. La aplicación tuvo una duración entre 30 y 45 minutos.

Para el análisis estadístico se utilizó el paquete estadístico SPSS versión 25.0. Se realizó inicialmente un análisis descriptivo univariado de los niveles de riesgo, ideación, depresión e impulsividad; una vez hecho esto se procedió a realizar un análisis bivariado con el fin de evaluar la asociación entre las distintas variables de estudio. Se utilizaron las pruebas no paramétricas de U de Mann-Whitney y Kruskal Wallis, debido a que en la aplicación de la prueba de Kolmogorov - Smirnov se encontró que las 
variables no se distribuyen de manera normal $(\mathrm{RS}=.137 ; \quad p<.001 ;$ PANSINEG $=.284 ; \quad p<.001$; PANSIPOS $=.146 ; \quad p<.001 ; \quad \mathrm{BDI}=.158 ; \quad p<.001$; $\mathrm{EI}=.081 ; p=.006)$. Posteriormente, se hizo el análisis de correlación mediante el coeficiente Rho de Spearman (Bull, Maslin, \& Armstrong, 2009). Finalmente, se realizó un análisis de ecuaciones estructurales con variables mediadoras para analizar cómo se afecta el riesgo suicida por las variables de impulsividad, depresión e ideación suicida. Para el modelado de ecuaciones estructurales se utilizó el software Amos versión 24.0. El tamaño del efecto ( $d$ Cohen) se calculó en R Studio Cloud.

\section{Resultados}

En la Tabla 1 se presentan los resultados arrojados por los instrumentos aplicados. El $20.7 \%$ de los adolescentes presenta riesgo suicida según la escala de Plutchik, cuya proporción corresponde al $21.3 \%$ al género femenino y el $19.7 \%$ al género masculino. En relación al inventario PANSI, el $12.8 \%$ presenta una ideación suicida negativa muy alta, de los cuales el $16.7 \%$ corresponden al género femenino y el $7 \%$ al género masculino, lo cual muestra que las mujeres presentan una mayor ideación suicida en comparación a los hombres. En cuanto a la ideación suicida positiva (factor protector), se evidencia que, el $84.9 \%$ presentan factores muy altos de protección en relación al suicidio. En la distribución por género, se muestra una diferencia leve en las mediciones en hombres (88.7\%) y mujeres $(82.4 \%)$. En la escala total, el $20.7 \%$ presentan un riesgo alto y el $9.5 \%$ presenta un riesgo muy alto. De las personas que presentan un riesgo total muy alto, el $12 \%$ corresponde al género femenino y el $5.6 \%$ al género femenino, lo cual muestra nuevamente un mayor riesgo en mujeres que en hombres.

Los resultados de la escala de impulsividad de Plutchik, muestran que el $22.3 \%$ presenta una tendencia a actuar y razonar de manera impulsiva, de los cuales el $26.9 \%$ son mujeres y el $15.5 \%$ hombres. En cuanto a la presencia de sintomatología depresiva de acuerdo al inventario de Beck, se identificó que el $2.8 \%$ presenta sintomatología grave, y el $17.3 \%$, síntomas moderados. El $61.5 \%$ no presenta sintomatología depresiva. En relación a la distribución no hay diferencias entre las medidas en hombres $(2.8 \%)$ y mujeres $(2.8 \%)$ para síntomas graves.

En relación a intentos previos de suicidio se encontró una tendencia baja, el $89.9 \%$ de los adolescentes indican no haber intentado suicidarse, mientras que el $9.5 \%$ si lo han intentado y de estos un 5\% lo han intentado una sola vez, un $1.1 \%$ dos veces, un $1.7 \%$ tres veces y un $1.8 \% 3$ o más veces. Respecto al tiempo se observa que un $3.3 \%$ lo intentó hace dos años, un $1.8 \%$ hace tres meses y un $0.6 \%$ hace dos semanas. El método más común de intento de suicidio es mediante el corte en las venas. En cuanto a la tendencia de intentos previos de suicidio discriminado por género, el $7.3 \%$ corresponde al género femenino y el $2.2 \%$ al género masculino, siendo más frecuente el intento de suicidio en mujeres que en hombres.

Tabla 1. Niveles de riesgo e ideación suicida, depresión e impulsividad

\begin{tabular}{|c|c|c|c|}
\hline Variable & Nivel & $\mathrm{n}$ & $\%$ \\
\hline \multirow{2}{*}{$\begin{array}{l}\text { Riesgo suicida } \\
\text { según escala de } \\
\text { Plutchik }\end{array}$} & Riesgo suicida & 37 & 20.7 \\
\hline & Sin riesgo suicida & 142 & 79.3 \\
\hline \multirow{3}{*}{$\begin{array}{l}\text { Ideación suicida } \\
\text { negativa }\end{array}$} & Bajo & 89 & 49.7 \\
\hline & Alto & 67 & 37.4 \\
\hline & Muy alto & 23 & 12.8 \\
\hline \multirow{5}{*}{$\begin{array}{l}\text { Ideación suicida } \\
\text { positiva }\end{array}$} & Muy bajo & 2 & 1.1 \\
\hline & Bajo & 3 & 1.7 \\
\hline & Medio & 6 & 3.4 \\
\hline & Alto & 16 & 8.9 \\
\hline & Muy alto & 152 & 84.9 \\
\hline \multirow{5}{*}{$\begin{array}{l}\text { Ideación suicida } \\
\text { total }\end{array}$} & Muy Bajo & 39 & 21.8 \\
\hline & Bajo & 48 & 26.8 \\
\hline & Medio & 38 & 21.2 \\
\hline & Alto & 37 & 20.7 \\
\hline & Muy Alto & 17 & 9.5 \\
\hline \multirow{2}{*}{$\begin{array}{l}\text { Impulsividad según } \\
\text { escala de Plutchik }\end{array}$} & Impulsividad & 40 & 22.3 \\
\hline & Sin impulsividad & 139 & 77.7 \\
\hline \multirow{5}{*}{$\begin{array}{l}\text { Inventario de } \\
\text { depresión de Beck }\end{array}$} & Valores perdidos & 3 & 1.7 \\
\hline & $\begin{array}{l}\text { Síntomas graves de } \\
\text { depresión }\end{array}$ & 5 & 2.8 \\
\hline & $\begin{array}{l}\text { Síntomas leves de } \\
\text { depresión }\end{array}$ & 30 & 16.8 \\
\hline & $\begin{array}{l}\text { Síntomas moderado de } \\
\text { depresión }\end{array}$ & 31 & 17.3 \\
\hline & Sin síntomas depresivos & 110 & 61.5 \\
\hline \multirow{3}{*}{$\begin{array}{l}\text { Intentos de suicidio } \\
\text { previo }\end{array}$} & No & 161 & 89.9 \\
\hline & No Responde & 1 & 6 \\
\hline & $\mathrm{Si}$ & 17 & 9.5 \\
\hline
\end{tabular}


Tabla 2. Riesgo e ideación suicida con impulsividad

\begin{tabular}{|c|c|c|c|c|c|c|}
\hline & Impulsividad & $n$ & Rango promedio & $z$ & $p$ & $d$ \\
\hline Ideación suicida neoativa & No & 139 & 82.03 & -3.913 & .000 & .6 \\
\hline & $\mathrm{Si}$ & 40 & 116.12 & -5.915 & .000 & .0 \\
\hline Ideación suicida positiva & $\begin{array}{c}\text { No } \\
\text { Si }\end{array}$ & $\begin{array}{c}139 \\
40\end{array}$ & $\begin{array}{l}91.67 \\
81.78\end{array}$ & -1.725 & .085 & .3 \\
\hline Ideación suicida total & $\begin{array}{c}\text { No } \\
\text { Si }\end{array}$ & $\begin{array}{c}139 \\
40\end{array}$ & $\begin{array}{c}84.68 \\
108.50\end{array}$ & -2.569 & .010 & .4 \\
\hline Riesgo suicida & $\begin{array}{c}\text { No } \\
\mathrm{Si}\end{array}$ & $\begin{array}{c}135 \\
37\end{array}$ & $\begin{array}{c}78.26 \\
116.58\end{array}$ & -4.204 & .000 & .7 \\
\hline
\end{tabular}

Tabla 3. Riesgo e ideación suicida y manifestaciones sintomáticas de depresión

\begin{tabular}{|c|c|c|c|c|c|c|}
\hline & Depresión & $n$ & $\begin{array}{c}\text { Rango } \\
\text { promedio }\end{array}$ & $H^{(g l)}$ & $p$ & $d$ \\
\hline \multirow{5}{*}{ Riesgo suicida } & Sin síntomas depresivos & 106 & 74.21 & \multirow{5}{*}{$51.310^{(3)}$} & \multirow{5}{*}{.000} & \multirow{5}{*}{1.2} \\
\hline & Síntomas leves de depresión & 30 & 93.67 & & & \\
\hline & Síntomas moderados de depresión & 29 & 106.17 & & & \\
\hline & Síntomas graves de depresión & 5 & 156.00 & & & \\
\hline & Valores perdidos & 9 & & & & \\
\hline \multirow{4}{*}{$\begin{array}{l}\text { Ideación suicida } \\
\text { negativa }\end{array}$} & Sin síntomas depresivos & 109 & 69.94 & \multirow{5}{*}{$50.594^{(3)}$} & \multirow{5}{*}{.000} & \multirow{5}{*}{1.2} \\
\hline & Síntomas leves de depresión & 30 & 106.33 & & & \\
\hline & Síntomas moderados de depresión & 31 & 122.77 & & & \\
\hline & Síntomas graves de depresión & 5 & 156.20 & & & \\
\hline \multirow{6}{*}{$\begin{array}{l}\text { Ideación suicida } \\
\text { positiva }\end{array}$} & Valores perdidos & 4 & & & & \\
\hline & Sin síntomas depresivos & 110 & 94.72 & \multirow{5}{*}{$20.730^{(3)}$} & \multirow{5}{*}{.000} & \multirow{5}{*}{0.7} \\
\hline & Síntomas leves de depresión & 30 & 83.65 & & & \\
\hline & Síntomas moderados de depresión & 30 & 74.70 & & & \\
\hline & Síntomas graves de depresión & 5 & 46.00 & & & \\
\hline & Valores perdidos & 4 & & & & \\
\hline \multirow{5}{*}{$\begin{array}{l}\text { Ideación suicida } \\
\text { total }\end{array}$} & Sin síntomas depresivos & 110 & 73.03 & \multirow{5}{*}{$36.286^{(3)}$} & \multirow{5}{*}{.000} & \multirow{5}{*}{1.0} \\
\hline & Síntomas leves de depresión & 30 & 97.43 & & & \\
\hline & Síntomas moderados de depresión & 31 & 122.40 & & & \\
\hline & Síntomas graves de depresión & 5 & 165.10 & & & \\
\hline & Valores perdidos & 3 & & & & \\
\hline
\end{tabular}

En cuanto a los estudiantes que presentaron impulsividad, se identificó que la ideación suicida negativa $(z=-3.913 ; p<.001 ; d=.6)$ y la ideación suicida total $(z=-2.569 ; p=.010 ; d=.4)$ son mayores respecto a los que no presentaron dicha característica. En ideación suicida positiva ( $\mathrm{z}=-1.725$; $p=.085)$ no hay diferencias significativas con relación a la impulsividad. Por otro lado, se observó que el riesgo suicida $(z=-4.204 ; p<.001$; $d=0.7$ ) es mayor en los estudiantes que presentaron rasgos de impulsividad en comparación a los que no. Al evaluar la $d$ de cohen se identificó un tamaño del efecto entre intermedio $(d>0.4)$ a grande $(\mathrm{d}>0.5)$, lo cual evidencia que las diferencias estadísticas encontradas son independientes del efecto del tamaño (ver Tabla 2).

En el análisis con la prueba de Kruskal Wallis se identificó que el riesgo suicida $(H=51.310$; $p<.001 ; d=1.2)$, la ideación suicida negativa $(H=50.594 ; p<.001 ; d=1.2)$ y la ideación suicida total $(H=36.286 ; p<.001 ; d=1.0)$ son mayores en los adolescentes que presentaron indicadores sintomáticos de depresión grave y moderada frente a los que no presentaron síntomas depresivos evaluados mediante el inventario de depresión de Beck. En cuanto a la ideación suicida positiva $(H=20.730 ; p<.001 ; d=.7)$ la relación es inversa en el sentido en que es mayor en los adolescentes que no evidencian síntomas depresivos en comparación a los que si presentan indicadores de depresión leve, moderada o grave. $\mathrm{Al}$ evaluar la $d$ de cohen se identificó un tamaño del efecto grande (d>.5) (ver Tabla 3).

En la Tabla 4 se muestran los resultados del análisis descriptivo y correlacional de las diversas variables de estudio. El riesgo suicida presenta una correlación significativa de signo positivo con la ideación suicida negativa ( $r=.649)$, ideación total $(r=.523)$, depresión ( $r=.725)$ e impulsividad global $(r=.444)$, y una correlación de signo negativo con la ideación suicida positiva ( $r=-.281)$. La ideación suicida negativa muestra correlaciones significativas con la depresión ( $r=.602)$ e ideación 
Tabla 4. Análisis descriptivo y correlacional de las variables de riesgo e ideación con impulsividad y

\begin{tabular}{lcccccccc}
\multicolumn{7}{c}{ depresión } \\
\hline & $\mathrm{M}$ & $\mathrm{D} . \mathrm{E}$ & 1 & 2 & 3 & 4 & 5 & 6 \\
\hline Riesgo suicida & 2.92 & 2.72 & 1 & & & & & \\
Ideación suicida negativa & 2.27 & 3.83 & $.649^{* *}$ & 1 & & & & \\
Ideación suicida positiva & 17.46 & 4.51 & $-.281^{* *}$ & $-.306^{* *}$ & 1 & & & \\
Depresión & 8.94 & 8.97 & $.725^{* *}$ & $.602^{* *}$ & $-.343^{* *}$ & 1 & & \\
Impulsividad & 14.95 & 6.46 & $.444^{* *}$ & $.371^{* *}$ & $-.155^{*}$ & $.339^{* *}$ & 1 & \\
Ideación suicida global & 9.28 & 7.60 & $.523^{* *}$ & $.669^{* *}$ & $-.868^{* *}$ & $.501^{* *}$ & $.299^{* *}$ & 1 \\
\hline
\end{tabular}

**. La correlación es significativa en el nivel .01 ( 2 colas).

*. La correlación es significativa en el nivel .05 ( 2 colas).

total ( $r=.669)$, y una correlación de signo negativo con la ideación suicida positiva $(r=-.306)$. En cuanto a la ideación suicida positiva, presenta una correlación significativa de signo negativo con la depresión ( $r=-.343)$, la impulsividad $(r=-.155)$ y la ideación suicida global ( $r=-.868)$. De otro lado, se evidenció una correlación fuerte entre la variable depresión y la impulsividad global $(r=.339)$.

En términos generales, se encontraron correlaciones estadísticamente significativas entre el riesgo y la ideación suicida negativa y global con la depresión y la impulsividad, mientras que, la ideación suicida positiva, la cual se considera un factor protector, presentó correlaciones de signo negativo con las variables de riesgo, ideación suicida global, depresión e impulsividad. De igual manera la depresión y la impulsividad presentaron una correlación positiva fuerte.

Con el fin de contrastar relaciones más complejas entre las variables consideradas y el riesgo suicida, no solo de causa y efecto, sino de relaciones explicativas entre las variables de respuesta y las variables independientes, se aplicó un análisis de ecuaciones estructurales con variables observables, a través de una variable mediadora, que para el caso del presente estudio, es la depresión, siguiendo la metodología propuesta en Baron y Kenny (1986). El modelo propuesto se presenta en la Figura 1. Con el fin de validar las relaciones que se plantean en este modelo se revisan por separado cada una de las relaciones directas e indirectas entre las variables dadas. Para este análisis se utilizó el procedimiento descrito en Hayes (2013) utilizando la macro PROCES 3.4 en SPSS versión 25. En este punto se observa que, considerando la depresión como variable de salida, las variables ideación suicida negativa, positiva e impulsividad, logran explicar un $41.9 \%$ de su variación $(\mathrm{F}(3,175)=42.098 ; p<$
$.001 ; d=1.6971)$. De igual manera, la ideación suicida negativa, la depresión, la ideación suicida positiva y la impulsividad, explican un $62.7 \%$ del riesgo suicida $(F(4,174)=73.1899 ; p<.001$; $d=2.5927$ ).

Con base en estos resultados, se procede a realizar la evaluación del modelo propuesto en la figura 1 y se establece el modelo de medida. Para establecer los indicadores de bondad de ajuste del modelo propuesto, se utiliza el método de estimación asymptotically distribution-free, bajo la condición de falta de normalidad multivariante de los datos (Byrne, 2013). El modelo obtenido presenta un muy buen ajuste absoluto $\left(X^{2}(2)=3.1\right.$; $p=.212>.05$ ), así mismo presenta un buen ajuste comparativo, lo cual se deduce a partir del índice de bondad de ajuste comparativo (CFI), el índice de ajuste normalizado (NFI) y el índice de Tucker - Lewis (CFI=.985>.90; NFI=.963>.90; TLI=.926>.90). También muestra un buen índice de bondad de ajuste (GFI) y un valor óptimo en la raíz del residuo cuadrático promedio (RMSEA) (GFI $=.987>.90 ; \quad$ RMSEA $=.056<.08)$. Estos resultados muestran que el modelo propuesto presenta un ajuste adecuado a los datos (McArdle, Nesselroade, McArdle, \& Nesselroade, 2014)

Por otro lado, los resultados muestran que el efecto total de las variables predictoras es de .4196, el cual, a partir del test de Sobel, resulta ser estadísticamente significativo $(t \quad(178)=9.8172$; $p<.001 ; \quad s e=.0427 ; \quad 95 \% \quad \mathrm{CI}[.1575, \quad .6024])$ (Holmbeck, 2002). Siguiendo la metodología planteada en Preacher \& Kelley (2011), se encuentra que la depresión tiene un porcentaje de mediación de $49.2 \%$ entre la impulsividad y el riesgo suicida (95\% CI [.1106,.2625]). Así mismo, esta misma variable presenta un porcentaje de mediación de $81.48 \%$ entre la ideación suicida negativa y el riesgo de suicidio (95\% CI [.0423,.0991]). Finalmente, resulta que la 


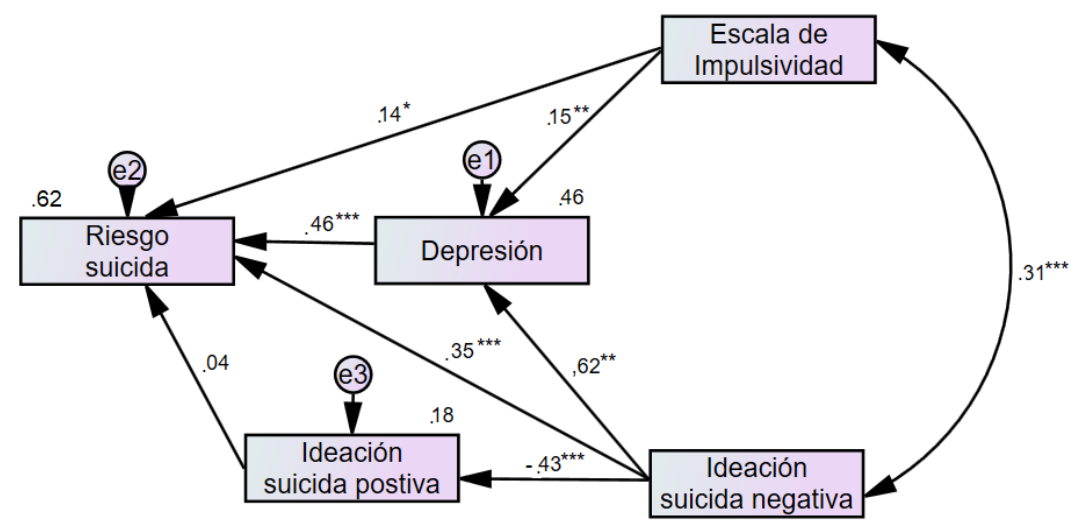

Figura 1. Modelo de ecuaciones estructurales: La depresión como variable mediadora $(* p<.05 ; * * p<.01, * * * p<.001)$

ideación suicida positiva, media un $4.9 \%$ entre la ideación suicida negativa y el riesgo suicida, pero esta última no resulta ser estadísticamente significativa (95\% IC [-.0492,.0730]).

\section{Discusión}

Considerando el objetivo del estudio realizado de establecer la relación entre el riesgo y la ideación suicida respecto de la impulsividad y la depresión en adolescentes, en este estudio se encontró una prevalencia de ideación suicida negativa del $50.2 \%$ y un factor general del $30.2 \%$, al igual que un indicador de riesgo del $20.7 \%$ en la población de adolescentes entre 14 y 17 años de edad. Este hallazgo en términos de la prevalencia de ideación y riesgo suicida está por encima a lo reportado en algunos estudios internacionales (Yoshimasu, Kiyohara, \& Miyashita, 2008), nacionales (Andrade \& Gonzáles, 2017; Siabato, Forero, \& Salamanca, 2017; Villalobos, 2009; Ceballos et al., 2015) y locales (Álvarez et al., 2011; Fuentes et al., 2009) en población de adolescentes escolares, lo cual indica que la población objeto de estudio presenta una prevalencia alta tanto para el factor de riesgo como el de ideación.

Al respecto, Villalobos (2009) y Siabato y Salamanca (2015), plantean que aproximadamente una cuarta parte de la población escolar presenta algún indicador de riesgo suicida e ideación suicida, entre ellos, pensamiento orientados a la muerte, ideación y fantasías de suicidio e indicadores relacionados con la depresión y la impulsividad, tal y como se demostró en este estudio y que es coincidente con otros estudios (Langhinrichsen-Rohling et al., 2009; Park et al., 2018). A pesar de que la prevalencia de intento de suicidio fue de $9.5 \%$, la ideación suicida se considera un predictor importante de la conducta suicida tanto para presentar o repetir una tentativa, como para el suicidio consumado en adolescentes y jóvenes (Siabato, Forero, \& Salamanca, 2017; Sánchez et al., 2005; Carmona et al., 2017; Carmona-Parra, \& Carmona-González, 2014), lo cual plantea la importancia de generar estrategias de prevención y abordaje psicosocial mucho antes de que se presente un intento o gesto suicida claro, lo cual se relaciona con un trabajo más centrado sobre factores protectivos (Collins et al., 2018).

El presente estudio permitió identificar que tanto la impulsividad como la depresión se relacionan de manera directa con el riesgo y la ideación suicida, de tal modo que, a mayor tendencia a la impulsividad y gravedad de los síntomas depresivos, mayor es el nivel de riesgo e ideación en los adolescentes estudiados. De acuerdo a Mann et al. (1999) y Oquendo, Galfalvy, Russo, Ellis y Mann (2004), la impulsividad y el bajo control inhibitorio reduce la autoeficacia emocional, especialmente del afecto negativo, lo cual se puede traducir en conductas de agresión auto dirigidas que pueden conducir al suicidio (David Klonsky, May, \& Glenn, 2013). Esto supone, de acuerdo a Siabato y Salamanca (2015) y Castañeda (2016) que existe una relación importante entre la conducta suicida y la reacción impulsiva que es necesario continuar 
estudiando en relación a su efecto en la comprensión del espectro suicida en la adolescencia, ya que los estudios no establecen aspectos concluyentes al respecto (Alasaarela et al., 2017; Auerbach et al., 2017; Franklin et al., 2017).

En este sentido, los principales factores de vulnerabilidad son la tendencia a actuar de manera irreflexiva, la impulsividad, la desesperanza y la depresión, entre otros factores biopsicosociales concomitantes que, en presencia de eventos vitales estresantes (Casullo \& Scheinsohn, 2006; Arenas-Landgrave et al., 2012; Madge et al., 2011; Thang, Li, Li, Wang, \& Siegrist, 2014), tales como problemas sociales, económicos, familiares, entre otros, aumentan dicha vulnerabilidad, y que puede desencadenar en un intento de suicidio (Bender, Gordon, Bresin, \& Joiner, 2011), en muchos casos acompañados de factores de riesgo en estilos de vida (Wilson, Fertuck, Kwitel, Stanley, \& Stanley, 2006) y aspectos de trastornos clínicos de fondo (Cipriani, Zhou, Del Giovane, Hetrick, Qin, \& Whittington et al., 2016; Horesh, Gothelf, Ofek, Weizman, \& Apter, 1999; Miché et al., 2018; Nock et al., 2009).

Diversas investigaciones (Oquendo, 2010; Salvo \& Melipillán, 2008; Villalobos, 2009a; Amezquita et al., 2008; Siabato \& salamanca, 2015), muestran que tanto la impulsividad como la depresión juegan un rol causal y predictor de la ideación y el comportamiento suicida en la adolescencia, lo cual se confirma en el presente estudio. El análisis de correlación y de predicción realizado demuestran justamente que tanto la variable de depresión como la de impulsividad presentan una relación estadísticamente significativa, además de ser variables predictores del riesgo y la ideación suicida, siendo la depresión la de mayor valor predictivo, aspecto similar en otros estudios (Alasaarela et al., 2017; Dougherty et al., 2009; Mortier, 2018; Wilkinson, Kelvin, Roberts, Dubicka, \& Goodyer, 2011).

En general, los hallazgos muestran un modelo de relaciones explicativas entre las variables, en el cual la depresión media entre las ideas suicida, la impulsividad y el riesgo, explicando un $41.9 \%$ la varianza del riesgo suicida. De igual manera, la ideación suicida negativa, la depresión, la ideación suicida positiva y la impulsividad, explican un $62.7 \%$ del riesgo suicida. Diversos estudios (Álvarez et al., 2013; Amezquita et al., 2008; Ceballos et al., 2015; Croarkin et al., 2018; Dumais et al., 2005; Hart et al., 2017; Nock et al., 2013; Mars et al., 2018), han enfatizado en el papel de la depresión y la ideación suicida en la predicción de la conducta suicida en adolescentes y jóvenes, estableciendo una relación de causa y efecto, sin embargo, no ha sido claro el rol de mediación de estas variables en la predicción del riesgo, lo cual es uno de los aportes más importante de este estudio, al encontrar que la depresión media entre la impulsividad y el riesgo de suicidio. Estos hallazgos corroboran la relación que tienen la depresión y la ideación suicida negativa como factores explicativos del riesgo de suicidio en adolescentes.

También se encontró que la ideación suicida positiva presenta una relación inversa con el riesgo de suicidio, dado que, como lo plantea Villalobos (2009a, b), esta variable se considera un factor protector ante el suicidio, asociado a la autoconfianza, sentido de control y satisfacción con la vida y la alegría y, por tanto, su relación con las demás variables es negativa (Langhinrichsen-Rohling et al., 2009).

Estos resultados son coherentes con el estudio realizado por Gonzales, Juárez, Montejo, Oaeguera, Wagner \& Jiménez, (2015), con población adolescente Mexicana, donde establece que factores como la depresión y la impulsividad explican, junto con el consumo de sustancias psicoactivas, el $44.2 \%$ de la ideación suicida; aspectos similares se ven en otros estudios internacionales (Borges, Benjet, Medina-Mora, Orozco, \& Nock, 2018; Franklin et al., 2017; Orri, Galera, Turecki, Forte, Renaud \& Boivin et al., 2018; Wilson et al., 2006). En estudios con población adolescente en Colombia también se encuentra consistencia en estos hallazgos (Amezquita et al., 2008; Siabato, \& Salamanca, 2015). De otro lado, la depresión ha demostrado ser uno de los principales predictores de la ideación y el intento suicida en la adolescencia. De acuerdo a Cañón et al. (2011), Amezquita et al. (2008), Carmona et al. (2017) y Ceballos et al. (2015), la depresión está asociada a los intentos de suicidio entre un 40 a un $70 \%$ de los casos en adolescentes escolares, $\mathrm{y}$ ha demostrado ser un predictor importante de la ideación suicida, de tal 
modo que, a mayor gravedad y persistencia de las manifestaciones depresivas mayor es la probabilidad de ocurrencia de una conducta suicida.

En cuanto al género, se identificó que las mujeres presentan un porcentaje mayor en ideación suicida negativa y global, al igual que en impulsividad, lo cual es coherente con lo encontrado por Villabos $\left(2009^{b}\right)$, Calvo, Sánchez, \& Tejada (2003), Amezquita et al. (2008) y Alvarez et al. (2012), quienes encontraron que el intento previo, el riesgo y la ideación suicida es más alto en población femenina, considerándose como un factor de riesgo en la adolescencia. Amezquita et al. (2008), en su estudio realizado en 8 colegios oficiales en la ciudad de Manizales, Colombia encontraron una prevalencia de ideación suicida de $45.3 \%$ y confirman que el género femenino y los altos niveles de depresión son los principales factores de riesgo suicida. Por otra parte, Álvarez et al. (2012) identifican un nivel de riesgo suicida mayor en adolescentes de género femenino siendo el porcentaje de riesgo de $17.9 \%$ en comparación con el género masculino de $5.3 \%$ en una institución educativa de Palestina, Caldas.

Finalmente, si bien la muestra del presente estudio no es alta, la tendencia de otros estudios internacionales muestra rasgos similares en sus resultados; este aspecto, permite visibilizar la necesidad de continuar indagando sobre los factores psicosociales asociados al riesgo y la ideación suicida, y especialmente su relación con la impulsividad en adolescentes escolares desde una perspectiva integradora y contextual, justamente porque no se presentan estudios concluyentes (Gvion et al., 2017; Franklin et al., 2017; Mortier et al., 2017; Mortier et al., 2018; Simon, Swann, Powell, Potter, Kresnow \& 'Carroll, 2002; Tan, Xia \& Reece, 2018; Yoshimasu et al., 2008). A partir de allí es donde se podrían diseñar estrategias focalizadas de prevención y atención en contextos educativos que respondan a las necesidades psicológicas y sociales de los adolescentes en la región, pues se considera que la previsibilidad debe ser el principal foco de atención y la investigación la herramienta que traza el camino sobre el cual se deben estructurar dichas estrategias.

\section{Referencias}

Aguirre, D. C., Cataño, J. J., Cañón, C., Marín, D. F., Rodríguez, J. T., Rosero, L. A., Valenzuela, L. P., \& Vélez, J. (2014). Riesgo suicida y factores asociados en adolescentes de tres colegios de la ciudad de Manizales (Colombia), 2013. Rev. Fac. Med, 63(3), 419-429. http://dx.doi.org/10.15446/revfacmed.v63n3.44 205

Alcázar, M. A., Bouso, J. C., \& Verdejo, A. J. (2015). Propiedades psicométricas de la escala de impulsividad de Plutchik en una muestra de jóvenes hispanohablantes. Actas Españolas de Psiquiatría, 43(5), 161-169. Recuperado de https://www.actaspsiquiatria.es/repositorio/17/ 97/ESP/17-97-ESP-161-9-505471.pdf

Álvarez, J. M., Cañón, S.C., Castaño, J. J., Bernier, L. H., Cataño, ... A. M., Galdino, (2013). Factor de riesgo suicida y factores asociados en adolescentes de una institución educativa de Palestina- Caldas (Colombia). Archivos de Medicina, 13(2), 127-141. Recuperado de http://www.redalyc.org/pdf/2738/2738297530 03.pdf

Alonso Polo, J., Castaño Castrillón, J., Ceron Rosero, Y., Dávila Mejura, L., de la Rosa, A., De la Rosa Marrugo, P., Montoya Arango, V., \& Olave Peña, C. (2015). Frecuencia de depresión, según cuestionario de beck, en estudiantes de medicina de la ciudad de Manizales (Colombia), 2014: Estudio de corte transversal. Archivos de Medicina, 15(1), 9$24 . \quad$ Recuperado de http://www.redalyc.org/pdf/2738/2738404350 02.pdf

Andrade, J., \& Gonzáles, J. (2017). Relación entre riesgo suicida, autoestima, desesperanza y estilos de socialización parental en estudiantes de bachillerato. Psicogente, 20(37), 70-88. http://doi.org/10.17081/psico.20.37.2419

Alasaarela, L., Hakko, H., Riala, K., \& Riipinen, P. (2017). Association of self-reported impulsivity to nonsuicidal self-injury, suicidality, and mortality in adolescent psychiatric inpatients. Journal of Nervous and Mental Disease, 205 (5), 340-345. doi:10.1097/NMD.0000000000000655 
Amezquita, M., Gonzales, R., \& Zuluaga, D. (2008). Prevalencia de depresión e ideación suicida en estudiantes de $8^{\circ}, 9^{\circ}, 10^{\circ}$ y $11^{\circ}$ grado, en ocho colegios oficiales de Manizales. Revista Hacia la Promoción de la Salud, 13, 143-153. Recuperado de: http://www.redalyc.org/html/3091/309126690 010/

Amézquita, M., González, R., \& Zuluaga, D. (2003). Prevalencia de la depresión, ansiedad y comportamiento suicida en la población estudiantil de pregrado de la Universidad de Caldas, año 2000. Revista Colombiana de Psiquiatría, 32(4), 341-356. Recuperado de http://www.redalyc.org/pdf/806/80632404.pdf

Auerbach, R. P., Stewart, J. G., \& Johnson, S. L. (2017). Impulsivity and suicidality in adolescent inpatients. Journal of Abnormal Child Psychology, 45(1), 91-103.

doi:10.1007/s10802-016-0146-8.

Arenas-Landgrave, P., \& Lucio-Gómez Marqueo, E., \& Forns, M. (2012). Indicadores diferenciales de personalidad frente al riesgo de suicidio en adolescentes. Revista Iberoamericana de Diagnóstico y Evaluación - e Avaliação Psicológica, 1(33), 51-74. Recuperado de http://www.redalyc.org/pdf/4596/4596454370 04.pdf

Baron, R. M., \& Kenny, D. A. (1986). The moderator-mediator variable distinction in social psychological research: Conceptual, strategic, and statistical considerations. Journal of Personality and Social Psychology, 51(6), 1173-1182. doi:10.1037/0022-3514.51.6.1173

Beck, A. T., Rush, A. J., Shaw, B. F., \& Emery, G. (1979). Cognitive therapy of depression. New York: Guilford Press (Trad. esp. en Bilbao: Desclée de Brower, 1983).

Bender, T. W., Gordon, K. H., Bresin, K., \& Joiner Jr, T.E. (2011). Impulsivity and suicidality: The mediating role of painful and provocative experiences. Journal of Affective Disorders, 129(1-3), 301-307. doi:10.1016/j.jad.2010.07.023

Borges, G., Benjet, C., Medina-Mora, M. E., Orozco, R., \& Nock, M. (2018). Suicide ideation, plan, and attempt in the Mexican adolescent mental health survey. Journal of the American Academy of Child and Adolescent Psychiatry, 47(1), 41-52. doi:10.1097/chi.0b013e31815896ad

Bull, F. C., Maslin, T. S., \& Armstrong, T. (2009). Global Physical Activity Questionnaire (GPAQ): Nine country reliability and validity study. Journal of Physical Activity and Health, 6(6), 790804. doi:10.1123/jpah.6.6.790

Byrne, B. M. (2013). Structural equation modeling with AMOS: Basic concepts, applications, and programming, second edition. New York: Routledge.

Calvo, J., Sánchez, R., \& Tejada, P. (2003). Prevalencia y factores asociados a ideación suicida en estudiantes universitarios. Revista Salud Pública, 5(2), 123-143. Recuperado de http://www.bdigital.unal.edu.co/21877/1/1840 6-61666-1-PB.pdf

Cañón, S. C., Castaño, J. J., Atehortúa, B. E., Botero, P., García, L. K., Rodríguez, L. M., Tovar, C. A., \& Rincón, E. (2012). Factor de riesgo para suicidio según dos cuestionarios y factores asociados en población estudiantil de la Universidad de Manizales (Colombia), 2011. Psicología desde el Caribe, 29(3), 632664. Recuperado de http://www.scielo.org.co/pdf/psdc/v29n3/v29 n3a05.pdf

Carpiniello, B., Lai, L., Pirarba, S., Sardu, C., \& Pinna, F. (2011). Impulsivity and aggressiveness in bipolar disorder with comorbid borderline personality disorder. Psychiatry Research, 188(1), 40-44. doi:10.1016/j.psychres.2010.10.026

Carmona, J. A, et al., (2017). El suicidio y otros comportamientos autodestructivos en jóvenes universitarios de Colombia y Puerto Rico: acciones, interacciones y significaciones. Manizales: Editorial Universidad de Manizales.

Carmona, J. A., \& Carmona, D. E. (2014). Intento de suicidio y variables relacionadas con la vida académica en estudiantes universitarios de la ciudad de Manizales. Revista Cultura del Cuidado, 11(2), 32-40. Recuperado de: http://repositorio.unilibrepereira.edu.co:8080/ Viewer/index.jsp?file $=123456789 / 356 / 3$ Suici dio.pdf 
Castaño, J. J., Cañón, S. C., Betancur, M., Castellanos, P. L., Guerrero, J., Gallego, A., \& Llanos, C. (2015). Factor de riesgo suicida según dos cuestionarios y factores asociados en estudiantes de la universidad nacional de Colombia sede Manizales. Diversitas: Perspectivas en Psicología, 11(2), 193-205. https://dx.doi.org/10.15332/s17949998.2015.0002.02

Castañeda, N. (2016). Prevención psicológica y neuropsicológica de factores de riesgo suicida en estudiantes universitarios. Psicogente, 19(36), 336-346. http://doi.org/10.17081/psico.19.36.1302

Casullo, M., \& Scheinsohn, M. (2006). Comportamientos suicidas en situaciones de internación psiquiátrica. Revista Iberoamericana de Diagnóstico y Evaluación - e Avaliação Psicológica, 2(22), 29-48. Disponible en http://www.aidep.org/03_ridep/R22/R222.pdf

Ceballos, G. A., Suarez, Y., Suescun, J., Gamarra, L. M., González, K. E., \& Sotelo, A. P. (2015). Ideación suicida, depresión y autoestima en adolescentes escolares de Santa Marta. Revista Internacional de Ciencias de la Salud, 12(1), 1522. http://dx.doi.org/10.21676/2389783X.1394

Cipriani, A., Zhou, X., Del Giovane, C., Hetrick, S.E., Qin, B., Whittington, C., et al ... (2016). Comparative efficacy and tolerability of antidepressants for major depressive disorder in children and adolescents: A network metaanalysis, The Lancet, 388(10047), 881-890. doi:10.1016/S0140-6736(16)30385-3

Coffin, N., Álvarez, M., \& Marín, A. (2011). Depresión e ideación suicida en estudiantes de la FESI: Un estudio piloto. Revista Electrónica de Psicología Iztacala, 14(4), 341-354. Recuperado de http://revistas.unam.mx/index.php/repi/article/ viewFile/28911/26864

Collins, K. R. L., Stritzke, W. G. K., Page, A. C., Brown, J. D., \& Wylde, T. J. (2018). Mind full of life: Does mindfulness confer resilience to suicide by increasing zest for life? Journal of Affective Disorders, 226, 100-107. doi:10.1016/j.jad.2017.09.043

Croarkin, P. E., Nakonezny, P. A., Deng, Z. D., Romanowicz, M., Voort, J. L. V., ... Camsari
(2018). High-frequency repetitive TMS for suicidal ideation in adolescents with depression. Journal of Affective Disorders, 239, 282-290. doi:10.1016/j.jad.2018.06.048

David Klonsky, E., May, A. M., \& Glenn, C. R. (2013). The relationship between nonsuicidal self-injury and attempted suicide: Converging evidence from four samples. Journal of Abnormal Psychology, 122(1), 231-237. doi:10.1037/a0030278

Dirección Territorial de Salud de Caldas. (2016). Informe comportamiento de los intentos de suicidio en el Departamento de Caldas año de 2016. Recuperado de: http://observatorio.saluddecaldas.gov.co/desca /anuales/Intento\%20de\%20Suicidio\%20a\%C3 $\%$ B1o\%202016.pdf

Dougherty, D. M., Mathias, C. W., MarshRichard, D. M., Prevette, K. N., Dawes, M. A., ... Hatzis, E. S. (2009). Impulsivity and clinical symptoms among adolescents with non-suicidal self-injury with or without attempted suicide. Psychiatry Research, 169(1), 22-27. doi: 10.1016/j.psychres.2008.06.011

Dumais, A., Lesage, A., Lalovic, A., Seguin, M., ... Turecki, G. (2005). Risk factors for suicide completion in major depression: A casecontrol study of impulsive and aggressive behaviors in men. Am J Psychiatry, 162(11), 2116-2124. Recuperado de https://pdfs.semanticscholar.org/f62c/9a7e6f4 b6cc9b503557f167c88ba6bebd7d0.pdf

Fernández Liporace, M., \& Casullo, M. (2006). Validación factorial de una escala para evaluar riesgo suicida. Revista Iberoamericana de Diagnóstico y Evaluación - e Avaliação Psicológica, 1(21), 9-22. Recuperado de http://www.redalyc.org/pdf/4596/4596454480 02.pdf

Franklin, J. C., Ribeiro, J. D., Fox, K. R., Bentley, K. H., Kleiman, E. M., ... Huang (2017). Risk factors for suicidal thoughts and behaviors: A meta-analysis of 50 years of research. Psychological Bulletin, 143(2), 187-232. doi:10.1037/bul0000084

Fuentes, M.M., Gonzales, A. F., Castaño, J.J., Hurtado, C. F., Ocampo, P. A., ... Páez, M. L., (2009). Riesgo suicida y factores de riesgo relacionados, en estudiantes de $6^{\circ}$ a $11^{\circ}$ grado 
en colegios de Manizales (Colombia) 20072008. Archivos de medicina, 9(2), 110-122. Recuperado de http://www.redalyc.org/html/2738/273820455 004/

Gómez, C., Rodríguez, N., Bohórquez, A., Díazgranados, N., Ospina, M. B., \& Fernández, C. (2002). Factores asociados al intento de suicidio en la población Colombiana. Revista Colombiana de Psiquiatría, 31(4), 271-283. Recuperado de http://www.scielo.org.co/pdf/rcp/v31n4/v31n4 a02.pdf

González, C., Juárez, C. E., Montejo, L. de los A., Oseguera, G., Wagner, F. A., \& Jiménez, A. (2015). Ideación suicida y su asociación con drogas, depresión e impulsividad en una muestra representativa de estudiantes de secundaria del estado de Campeche, México. Acta Universitaria, 25(NE-2), 29-34. doi:10.15174/au.2015.862

Gutiérrez, A., Contreras, C. M., \& Orozco, R.C. (2006). El suicidio, conceptos actuales. Salud mental, 29(5), 66-74. Recuperado de http://www.inprfcd.gob.mx/pdf/sm2905/sm290566.pdf

Gvion, Y., \& Apte, A. (2011). Aggression, impulsivity and suicide behavior: A review of the literatura. Archives of Suicide Research, 15(2), 93-112. doi:10.1080/13811118.2011.565265

Hart, S. R., Van Eck, K., Ballard, E. D., Musci, R. J., Newcomer, A., \& Wilcox, H. C. (2017). Subtypes of suicide attempters based on longitudinal childhood profiles of cooccurring depressive, anxious and aggressive behavior symptoms. Psychiatry Research, 257 , 150-155. https://doi.org/10.1016/j.psychres.2017.07.032

Hayes, A. (2013). Introduction to mediation, moderation, and conditional process analysis. New York: The Guilford Press

Hernández Sampieri, R. Collado, C. F., \& Baptista, P. (2014). Metodología de la investigación. 6 ediciones. México: McGRAW-HILL.

Horesh, N., Gothelf, D., Ofek, H., Weizman, T., \& Apter, A. (1999). Impulsivity as a correlate of suicidal behavior in adolescent psychiatric inpatients. Crisis, 20(1), 8-14. doi: 10.1027//0227-5910.20.1.8

Holmbeck, G. N. (2002). Post-hoc probing of significant moderational and mediational effects in studies of pediatric populations. Journal of Pediatric Psychology, 27(1), 8796. doi:10.1093/jpepsy/27.1.87

Instituro Nacional de Medicina Legal y Ciencias Forenses (2016). 2016 FORENSIS datos para la vida. Recuperado de http://www.acotaph.org/assets/forensis-2016.pdf

Langhinrichsen-Rohling, J., Friend, J., \& Powell, A. (2009). Adolescent suicide, gender, and culture: A rate and risk factor analysis. Aggression and Violent Behavior, 14(5), 402414. doi:10.1016/j.avb.2009.06.010

Madge, N., Hawton, K., McMahon, E.M., Corcoran, P., De Leo, ... D., De Wilde (2011). Psychological characteristics, stressful life events and deliberate self-harm: Findings from the child \& adolescent self-harm in Europe (CASE) study. European Child and Adolescent Psychiatry, 20(10), 499-508. doi:10.1007/s00787-011-0210-4

Mann, J. J., Oquendo, M., Underwood, M. D., \& Arango V. (1999). The neurobiology of suicide risk: A review for the clinician. Journal Clinical Psychiatry, 60(2), 7-11. Recuperado de https://psycnet.apa.org/record/1999-10282002

Mars, B., Heron, J., Klonsky, E. D., Moran, P., O'Connor, R. C., Tilling, K., ... Gunnell, D. (2018). What distinguishes adolescents with suicidal thoughts from those who have attempted suicide? A population-based birth cohort study. Journal of Child Psychology and Psychiatry. https://doi.org/10.1111/jcpp.12878

McArdle, J. J., \& Nesselroade, J. R. (2014). Basics of structural equation modeling. In J. J. McArdle \& J. R. Nesselroade, Longitudinal data analysis using structural equation models (pp. 27-37). Washington, DC, US: American Psychological Association.

Miché, M., Hofer, P. D., Voss, C., Meyer, A. H., Gloster, A. T., Beesdo-Baum, K., \& Lieb, R. (2018). Mental disorders and the risk for the subsequent first suicide attempt: Results of a community study on adolescents and young 
adults. European Child and Adolescent Psychiatry, 27(7), 839-848. doi:10.1007/s00787-017-1060-5

Molina, Y., Gómez, O., Pabòn, J., Ropero, U., Vélez, D., \& Salazar, J. F. (2018). Prevalencia de sintomatología depresiva en estudiantes de medicina de la Universidad de Caldas, Manizales - Colombia. Rev. Méd. Risaralda, 24(1), 23-28.

Mortier, P., Auerbach, R.P., Alonso, J., Bantjes, J., Benjet, C., ... Cuijpers, P. (2018). Suicidal thoughts and behaviors among first-year college students: Results From the WMH-ICS Project. Journal of the American Academy of Child and Adolescent Psychiatry, 57(4), 263273. doi:10.1016/j.jaac.2018.01.018

Mortier, P., Kiekens, G., Auerbach, R. P., Cuijpers, P., Demyttenaere, ... K., Green (2017). A risk algorithm for the persistence of suicidal thoughts and behaviors during college. Journal of Clinical Psychiatry, 78(7), 828-836. doi:10.4088/JCP.17m1 1485

Nock, M. K., Hwang, I., Sampson, N., Kessler, R. C., Angermeyer, ... M., Beautrais (2009). Cross-national analysis of the associations among mental disorders and suicidal behavior: Findings from the WHO World Mental Health Surveys. PLoS Medicine, 6 (8). doi:10.1371/journal.pmed.1000123

Nock, M. K., Green, J. G., Hwang, I., McLaughlin, K. A., Sampson, N. A., Zaslavsky, A. M., \& Kessler, R. C. (2013). Prevalence, correlates, and treatment of lifetime suicidal behavior among adolescents. JAMA Psychiatry, 70(3), 300. https://doi.org/10.1001/2013.jamapsychiatry.5 5

Oquendo, M. (2010) predictores clínicos de conducta suicida. Abordaje prospectivo (tesis doctoral). Universidad Autónoma de Madrid, España. Recuperado de https://repositorio.uam.es/bitstream/handle/10 486/5658/35434_oquendo_villar_maria_anton ia.pdf? sequence $=1$

Oquendo, M. A., Galfalvy, H., Russo, S., Ellis, S., \& Mann, J. J. (2004). Prospective Study of clinical predictors of suicidal acts after a major depressive episode in patients with major depressive disorder or bipolar disorder.
Am Journal Psychiatry, 161(14), 33-41. doi:10.1176/appi.ajp.161.8.1433

Organización Mundial de la Salud (2017). Depresión, Recuperado de http://www.who.int/topics/depression/es/

Organización Mundial de la Salud (2017). Suicidio. Recuperado de http://www.who.int/mediacentre/factsheets/fs 398/es/.

Osman, A., Barrios, F., Gutiérrez, P., Wrangham, J., Kopper, B., Truelove, R., \& Linden, S. (2002). The Positive and Negative Suicide Ideation Inventory (PANSI): Psychometric evaluation with adolescent psychiatric inpatient samples. Journal of Personality Assessment, $\quad 79(3), \quad$ 512-530. https://doi.org/10.1207/S15327752JPA7903_0 7

Orri, M., Galera, C., Turecki, G., Forte, A., Renaud, J., Boivin, M., Tremblay, R. E., Cote, S. M., \& Geoffroy, M. C. (2018). Association of childhood irritability and depressive/anxious mood profiles with adolescent suicidal ideation and attempts. JAMA Psychiatry, 75(5), 465-473. doi:10.1001/jamapsychiatry.2018.017

Park, S., \& Jang, H. (2018). Correlations between suicide rates and the prevalence of suicide risk factors among Korean adolescents. Psychiatry Research, 261, 143-147. doi:10.1016/j.psychres.2017.12.055

Plutchik, R., \& Van Praag, H. M. (1989). The measurement of suicidality, aggressivity and impulsivity. Progress in NeuroPsychopharmacology \& Biological Psychiatry, 13, 23-24.

Delgado, L. P., Jaramillo, D. P., Nieto, E., Saldarriaga, G. I., Giraldo, C. L., ... Orozco, M. I. (2017). Política Pública de Salud Mental del departamento de Caldas: Un aporte al bienestar y a la inclusión. Manizales: Editorial Universidad Autónoma de Manizales

Riaño-Hernández, D., Guillen, A., \& Buela-Casal, G. (2015). Conceptualización y evaluación de la impulsividad en adolescentes: Una revisión sistemática. Universitas Psychologica, 14(3), 1077-1090. http://dx.doi.org/10.11144/Javeriana.upsy143.ceia 
Rubio, G., Montero, I., Jáuregui, J., Villanueva, R., Casado, M. A., Marín, J. J., \& SantoDomingo, J. (1998). Validación de la escala de riesgo suicida de Plutchik en población española. Archivos de Neurobiología, 61(2), 143-52.

Rubio, G., Montero, I., Jáureguir, J., Martínez, M. L., Álvarez, S., Marín, J. J. et al. (1999). Validación de la Escala de Impulsividad de Plutchik en población española. Archivos de Neurobiología, 61, 223-232.

Rueda, G. H., Rangel, M. A., Castro, V. A., \& Camacho, P. A. (2010). Suicidabilidad en adolescentes, una comparación con población adulta. Revista Colombiana de Psiquiatría. 39(4), 683-692. Recuperado de http://www.redalyc.org/html/806/8061923100 3/

Salvo, L., \& Melipillán, R. (2008). Predictores de suicidalidad en adolescentes. Revista Chilena de Neuropsiquiatría, 46(2), 115-123. http://dx.doi.org/10.4067/S071792272008000200005

Sánchez, R., Guzmán, Y., \& Cáceres, H. (2005). Estudio de la imitación como factor de riesgo para ideación suicida en estudiantes universitarios adolescentes. Revista Colombiana de Psiquiatría, 34(1), 12-25. Recuperado de http://www.redalyc.org/html/806/8062840300 3/

Sanz, J., \& Vázquez, C. (1998). Fiabilidad, validez y datos normativos del Inventario para la Depresión de Beck. Psicothema, 10(2), 303-318. Recuperado de http://www.psicothema.com/pdf/167.pdf

Shang, L., Li, J., Li, Y., Wang, T., \& Siegrist, J. (2014). Stressful psychosocial school environment and suicidal ideation in Chinese adolescents. Social Psychiatry and Psychiatric Epidemiology, 49(2), 205-210. doi:10.1007/s00127-013-0728-5

Siabato, E., \& Salamanca, Y. (2015). Factores asociados a ideación suicida en universitarios. Psychologia: Avances de la Disciplina, 9(1), 71-81. Recuperado de: http://www.scielo.org.co/pdf/psych/v9n1/v9n $1 \mathrm{a} 06 . p d f$

Siabato, E., Forero, I. X., \& Salamanca, Y. (2017). Asociación entre depresión e ideación suicida en un grupo de adolescentes colombianos. Pensamiento Psicológico, 15(1), 5161.doi:10.11144/Javerianacali.PPSI15-1.ADIS.

Simon, T. R., Swann, A. C., Powell, K. E., Potter, L. B., Kresnow, M. J., \& O'Carroll, P. W. (2002). Characteristics of impulsive suicide attempts and attempters. Suicide and LifeThreatening Behavior, 32(1 SUPPL.), 49-59.

Tan, L., Xia, T., \& Reece, C. (2018). Social and individual risk factors for suicide ideation among Chinese children and adolescents: A multilevel analysis. International Journal of Psychology, 53(2),

117-125. doi:10.1002/ijop.12273

Téllez J., \& Forero, J. (Ed). (2006). Suicidio: Neurobiología, factores de riesgo y prevención. Bogotá: Editorial Nuevo Milenio.

Villalobos, F. H. (2009a) Situación de la conducta suicida en estudiantes de colegios y universidades de San Juan de Pasto, Colombia. Salud Mental, 32(2), 165-171. Recuperado de: http://www.scielo.org.mx/scielo.php?script=sc i_arttext\&pid=S0185-

$33252009000200009 \& \operatorname{lng}=\mathrm{es} \& \operatorname{tlng}=\mathrm{es}$.

Villalobos, F. H. (2009b). Validez y fiabilidad del Inventario de Ideación Suicida Positiva y Negativa - PANSI, en estudiantes colombianos. Universitas Psychologica, 9(2), 509-520. Recuperado de http://www.scielo.org.co/pdf/rups/v9n2/v9n2a 17.pdf

Wilkinson, P., Kelvin, R., Roberts, C., Dubicka, B., \& Goodyer, I. (2011). Clinical and psychosocial predictors of suicide attempts and nonsuicidal self-injury in the Adolescent Depression Antidepressants and Psychotherapy Trial (ADAPT). American Journal of Psychiatry, 168(5), 495-501. doi:10.1176/appi.ajp.2010.10050718

Wilson, S. T., Fertuck, E. A., Kwitel, A., Stanley, M. C., \& Stanley, B. (2006). Impulsivity, suicidality and alcohol use disorders in adolescents and young adults with borderline personality disorder. International Journal of Adolescent Medicine and Health, 18(1), 189196. doi:10.1515/IJAMH.2006.18.1.189

Witte, T. K., Merrill, K. A., Stellrecht, N. E., Bernert, R. A., Hollar, D. L., Schatschneider, C., \& Joiner Jr., T. E. (2008). "Impulsive" 
youth suicide attempters are not necessarily all that impulsive. Journal of Affective Disorders, 107(1-3), 107-116. doi:10.1016/j.jad.2007.08.010

Yoshimasu, K., Kiyohara, C., \& Miyashita, K. (2008). Suicidal risk factors and completed suicide: Meta-analyses based on psychological autopsy studies. Environmental Health and Preventive Medicine, 13(5), 243256. doi:10.1007/s12199-008-0037-x 